\title{
A Diagnostic Score for Acute Small Bowel Obstruction
}

\author{
MAARET ESKELINEN ${ }^{1 *}$, JANNICA MEKLIN $^{1 *}$, KARI SYRJÄNEN $^{2,3}$ and MATTI ESKELINEN ${ }^{1}$ \\ ${ }^{1}$ Department of Surgery, Kuopio University Hospital and School of Medicine, \\ University of Eastern Finland, Kuopio, Finland; \\ ${ }^{2}$ Molecular Oncology Research Center, Barretos Cancer Hospital, Barretos, Brazil; \\ ${ }^{3}$ SMW Consultants, Ltd., Kaarina, Finland
}

\begin{abstract}
Background/Aim: The diagnosis of acute small bowel obstruction (ASBO) may be difficult and the decision to operate is based on clinical findings. So far, the diagnostic scores (DSs) for ASBO detection have been rarely evaluated. Patients and Methods: A cohort of 1,333 acute abdominal pain (AAP) patients with 54 ASBO patients, were included in the study. The most significant diagnostic findings (in multivariate logistic regression analysis) were used to construct DS formulas for ASBO diagnosis with location of pain at diagnosis $(\mathrm{LP}+)$ and without location of pain at diagnosis (LP-). Metaanalytical techniques were used to calculate the summary sensitivity ( $\mathrm{Se}$ ) and specificity ( $\mathrm{Sp}$ ) estimates for each data sets (history-taking, findings, and DS formulas). Results: In SROC analysis, the AUC values for $i)$ clinical history-taking, ii) diagnostic findings and tests, iii) $D S_{L P-}$ and $\left.i v\right) D S_{L P+}$ were as follows: i) $A U C=0.638$ (95\%CI=0.600-0.676); ii) $A U C=0.694$ (95\%CI=0.630-0.724), iii) $A U C=0.962$ (95\% CI=0.940-0.986), and for $i v) A U C=0.971$ (95\%CI=0.952-0.988). In roccomp analysis for the AUC values, the differences are significant as follows: between i) and ii) $p=0.312$; between i) and iii) $p<0.0001$; between i) and iv) $p<0.0001$; between ii) and iii) $p<0.0001$; between ii) and iv) $p<0.0001$; and between iii) and iv) $p=0.317$. Conclusion: The present study is the first to provide data that the DS could be used for clinical diagnosis of ASBO without radiological or laboratory analyses, to reach a high diagnostic accuracy in AAP patients.
\end{abstract}

This article is freely accessible online.

*These Authors contributed equally to this study.

Correspondence to: Matti Eskelinen, MD, Ph.D., School of Medicine, University of Eastern Finland, P.O. Box 100, FI-70029 KYS, Finland. Tel: +358 17173311, Fax: +358 17172611, GSM: +358 400969444, e-mail: matti.eskelinen@kuh.fi

Key Words: Acute small bowel obstruction, diagnostic score, ROC, HSROC, diagnostic accuracy.
Although the diagnosis of acute small bowel obstruction (ASBO) is sometimes difficult, the diagnosis and treatment of ASBO is necessary for intestinal preservation and avoiding the risk of complications. The clinical findings of ASBO include signs of peritoneal irritation and the acute abdominal pain (AAP) with distension, abnormal bowel sounds and a history of abdominal surgery, because adhesions are complications of abdominal surgery and the most frequent etiology of $\operatorname{ASBO}(1,2)$. However, it is difficult to diagnose ASBO based on a single history-taking variable or a clinical finding. Physical examination of the patient is thus important for making the decision to operate or not. In practice, the decision of operation in patients with ASBO is based on doctor's clinical judgement. The lack of commonly accepted guidelines and diagnostic scoring (DS) for ASBO (1) encouraged us to evaluate the accuracy of the clinical diagnosis of ASBO among AAP patients. We designed the present study to assess the relative accuracy of i) a clinical history-taking, ii) clinical findings, as well as iii) the DS without location of pain at diagnosis ( $\mathrm{LP}-$ ) and iv) the DS with location of pain at diagnosis (LP+) in confirming ASBO among the patients with AAP.

\section{Patients and Methods}

A cohort of 1333 acute abdominal pain (AAP) patients with 54 ASBO patients was Included in the present. The clinical symptoms $(n=22)$, signs $(n=14)$ and laboratory tests $(n=3)$ were recorded for each patient. The diagnosis of ASBO was confirmed by considering all clinical history-taking details, clinical findings and results of the laboratory tests and following the diagnostic criteria of ASBO as previously described (3-7) (Tables I and II).

Identifying the DS models. A multivariate logistic (stepwise) regression analysis (SPSS Statistics 26.0.0.1; IBM, Armonk, NY, USA) was used to disclose the variables with an independent predictive value. All the variables of clinical history and diagnostic findings presented in Tables I and II were included in the analysis as binary data e.g. ASBO $=1$ and other diagnosis of $\mathrm{AAP}=0$. Using the coefficients of the regression model, a DS was built and its predictive value for ASBO was studied. The coefficient of the multivariate analysis shows the relative risk 
Table I. The clinical history of the acute small bowel obstruction (ASBO) patients versus other causes of abdominal pain.

\begin{tabular}{|c|c|c|c|c|c|c|}
\hline Clinical history variable & Positive endpoint & Negative endpoint & $\mathrm{TP}$ & FN & FP & $\mathrm{TN}$ \\
\hline 1. Location of initial pain & Upper, center, lower abdomen, general & Other quadrants of the abdomen & 47 & 7 & 761 & 518 \\
\hline 2. Location of pain at diagnosis & Upper, center, lower abdomen, general & Other quadrants of abdomen & 42 & 12 & 498 & 781 \\
\hline $\begin{array}{l}\text { 3. Duration of pain: Duration } \\
\text { of pain at diagnosis }\end{array}$ & $\leq 6$ hours & $>6$ hours & 26 & 28 & 435 & 844 \\
\hline 4. Intensity of abdominal pain & Subjectively moderate or intolerable pain & Weak pain & 43 & 11 & 830 & 449 \\
\hline $\begin{array}{l}\text { 5. Progression of pain from } \\
\text { onset to diagnosis }\end{array}$ & Subjectively same or worse pain & Weaker pain than at the onset & 42 & 12 & 846 & 433 \\
\hline 6. Type of pain & Subjectively colicky or Intermitted pain & Steady pain & 37 & 17 & 565 & 714 \\
\hline 7. Aggravating factors & No aggravating factors & $\begin{array}{l}\text { Movement, coughing, respiration, } \\
\text { food or other }\end{array}$ & 20 & 34 & 336 & 943 \\
\hline 8. Relieving factors & Vomiting & $\begin{array}{l}\text { Lying still, food, antacids or } \\
\text { no relieving factors }\end{array}$ & 10 & 44 & 88 & 1,091 \\
\hline 9. Previous similar pain & Yes & No & 21 & 32 & 426 & 840 \\
\hline 10. Vertigo & Yes & No & 4 & 50 & 36 & 1,239 \\
\hline 11. Nausea & Yes & No & 43 & 11 & 723 & 556 \\
\hline 12. Vomiting & Yes & No & 37 & 17 & 533 & 746 \\
\hline 13. Appetite & No appetite & Normal appetite & 50 & 4 & 927 & 352 \\
\hline 14. Previous indigestion & Yes & No & 18 & 36 & 261 & 1,016 \\
\hline 15. Jaundice & No & Yes & 53 & 1 & 1,247 & 30 \\
\hline 16. Bowels & Constipation & $\begin{array}{l}\text { Diarrhea, blood, mucus, white } \\
\text { or normal stools }\end{array}$ & 20 & 34 & 126 & 1,153 \\
\hline 17. Micturition & Normal & Abnormal & 52 & 2 & 1,195 & 84 \\
\hline 18. Drugs for abdominal pain & Yes & No & 4 & 50 & 50 & 1,228 \\
\hline 19. Previous abdominal surgery & Yes & No & 46 & 8 & 287 & 991 \\
\hline 20. Previous abdominal diseases & Yes & No & 19 & 35 & 214 & 1,063 \\
\hline 21. Use of alcohol & No & Yes & 1 & 53 & 1,212 & 66 \\
\hline
\end{tabular}

$(\mathrm{RR}=\mathrm{en}, \mathrm{n}=ß)$ of a patient with a given history-taking detail, clinical finding or test to have an ASBO.

The DS formula for ASBO without location of pain ( $L P-$-). DS: 0.83 $\mathrm{x}$ type of pain (positive endpoint $=1$, negative endpoint $=0$ ) $+2.45 \times$ previous abdominal surgery (positive endpoint $=1$, negative endpoint $=0)+2.79 \times$ distension (positive endpoint $=1$, negative endpoint $=0)+2.25 \times$ bowel sounds (positive endpoint $=1$, negative endpoint $=0)-3.85$ (Table III).

The DS formula for ASBO with location of pain (LP+). DS: $1.03 \times$ location of pain at diagnosis (positive endpoint=1, negative endpoint $=0$ ) $+0.87 \times$ type of pain (positive endpoint $=1$, negative endpoint $=0$ ) $+2.50 \times$ previous abdominal surgery (positive endpoint $=1$, negative endpoint $=0$ ) $+2.57 \times$ distension (positive endpoint $=1$, negative endpoint $=0$ ) $+2.20 \times$ bowel sounds (positive endpoint $=1$, negative endpoint $=0)-7.22($ Table IV).

Statistical analysis. The other statistical analyses were performed using STATA/SE version 16.1 (StataCorp, College Station, TX, USA). Statistical tests presented were two-sided, and $p$-value $<0.05$ was considered statistically significant. Using $2 \times 2$ tables, we calculated sensitivity (Se) and specificity ( $\mathrm{Sp}$ ) with $95 \%$ confidence intervals $(95 \% \mathrm{CI})$ for each clinical history-taking detail, finding or test, and using a meta-analytical technique (metaprop) separate forest plots for $\mathrm{Se}$ and $\mathrm{Sp}$ were created for each set of data, including each diagnostic variable (as equivalent to "study ID"). We calculated the summary estimates of Se and $\mathrm{Sp}$, positive (LR+) and negative likelihood ratio (LR-) and diagnostic odds ratio (DOR), using a random effect bivariate model and fitted the summary hierarchical receiving operating characteristic (HSROC) curves, including all diagnostic variables in the $\mathrm{DS}_{\mathrm{LP}-}$ and $\mathrm{DS}_{\mathrm{LP}+}$ models, using the ASBO endpoint.

\section{Results}

The clinical history-taking in ASBO. The overall Se of the clinical history-taking for detecting ASBO was $57 \%$ (95\% CI $=41-72 \%)$. Se was higher than $57 \%$ for 11 symptoms. The five best clinical history-taking variables (location of initial pain, appetite, jaundice, micturition, previous abdominal surgery) showed $85-98 \%$ Se in diagnosis of ASBO (Figure 1). The Sp of the history-taking for detecting ASBO was 57\% (95\%CI=42-72\%) (Figure 2). Altogether, 12 symptoms showed Sp higher than 57\%. The five best symptoms of ASBO (relieving factors, vertigo, previous indigestion, bowels, drugs for abdominal pain) showed 83-93\% Sp (Figure 2).

The clinical findings and tests in ASBO. The overall Se of the diagnostic findings for ASBO was 75\% (95\%CI=62$86 \%$ ) (Figure 3), and 10 findings had Se exceeding 75\%. The five most accurate findings (colour, scar, mass, Murphy's 
Table II. The clinical signs and tests of the acute small bowel obstruction (ASBO) patients versus those with other causes of abdominal pain.

\begin{tabular}{|c|c|c|c|c|c|c|}
\hline Clinical signs and investigations & Positive endpoint & Negative endpoint & $\mathrm{TP}$ & FN & FP & $\mathrm{TN}$ \\
\hline 1. Mood & Distressed or anxious & Normal & 14 & 40 & 213 & 1,066 \\
\hline 2. Colour & Normal & Jaundiced, pale, flushed or cyanosed & 48 & 6 & 1,133 & 146 \\
\hline 3. Abdominal movement & Poor/nil & Normal & 10 & 44 & 83 & 1,195 \\
\hline 4. Scar & Yes & No & 46 & 8 & 300 & 978 \\
\hline 5. Distension & Yes & No & 36 & 18 & 57 & 1,218 \\
\hline 6. Tenderness & Upper, center, lower abdomen, general & Other quadrants of the abdomen & 37 & 17 & 342 & 937 \\
\hline 7. Mass & No & Yes & 54 & 0 & 1,245 & 34 \\
\hline 8. Rebound & No & Yes & 32 & 22 & 670 & 609 \\
\hline 9. Guarding & Yes & No & 34 & 20 & 673 & 606 \\
\hline 10. Rigidity & No & Yes & 45 & 9 & 993 & 285 \\
\hline 11. Murphy's positive & No & Yes & 53 & 1 & 1,155 & 123 \\
\hline 12. Bowel sounds & Abnormal & Normal & 41 & 13 & 148 & 1,131 \\
\hline 13. Renal tenderness & No & Yes & 44 & 10 & 928 & 351 \\
\hline 14. Rectal digital tenderness & Normal & Abnormal & 43 & 11 & 926 & 353 \\
\hline 15. Body temperature & $\leq 37.1^{\circ} \mathrm{C}$ & $>37.1^{\circ} \mathrm{C}$ & 39 & 10 & 667 & 516 \\
\hline 16. Leucocyte count (LC) & $>10,000 / \mathrm{mm} 3$ & $\leq 10,000 / \mathrm{mm}^{3}$ & 18 & 22 & 447 & 594 \\
\hline 17. Urine & Normal & Haematuria or bacteriuria & 42 & 0 & 1,054 & 72 \\
\hline
\end{tabular}

TP: True positive; FN: false negative; FP: false positive; TN: true negative.

Table III. Diagnostic score for acute small bowel obstruction (ASBO) patients without 'location of pain at diagnosis' in the DS model (DS $\left.L_{L P-}\right)$. The DS model is shown at six different cut-off levels of symptoms, signs and tests. Cut-off levels: DS I=-3.85, DS II=-3.56, DS III=-1.94, DS IV=-1.60, $D S V=D S$ values between -3.85 and -1.60 excluded, $n=188$ patients.

\begin{tabular}{|c|c|c|c|c|c|c|}
\hline Diagnostic score (DS) & Positive endpoint & Negative endpoint & $\mathrm{TP}$ & FN & FP & $\mathrm{TN}$ \\
\hline 1. Logistic model $\mathrm{DS}_{\mathrm{LP}-\mathrm{I}}$ & ASBO & Other cause of abdominal pain & 51 & 3 & 234 & 1,040 \\
\hline 2. Logistic model $\mathrm{DS}_{\mathrm{LP}}$ II & ASBO & Other cause of abdominal pain & 49 & 5 & 181 & 1,093 \\
\hline 3. Logistic model $\mathrm{DS}_{\mathrm{LP}-}$ III & ASBO & Other cause of abdominal pain & 47 & 7 & 68 & 1,206 \\
\hline 4. Logistic model $\mathrm{DS}_{\mathrm{LP}}$ IV & ASBO & Other cause of abdominal pain & 43 & 11 & 54 & 1,220 \\
\hline 5. Logistic model $\mathrm{DS}_{\mathrm{LP}} \mathrm{V}$ & ASBO & Other cause of abdominal pain & 43 & 3 & 54 & 1,040 \\
\hline
\end{tabular}

TP: True positive; FN: false negative; FP: false positive; TN: true negative. Logistic regression analysis formula for DS: $0.83 \times$ type of pain (positive endpoint $=1$, negative endpoint $=0)+2.45 \times$ previous abdominal surgery (positive endpoint $=1$, negative endpoint $=0)+2.79 \times$ distension $($ positive endpoint $=1$, negative endpoint $=0)+2.25 \times$ bowel sounds (positive endpoint $=1$, negative endpoint $=0)-3.85$.

Table IV. Diagnostic score for acute small bowel obstruction (ASBO) patients with 'location of pain at diagnosis' in the DS model (DS $\left.L_{L+}\right)$. The $D S$ model is shown at five different cut-off levels of symptoms, signs and tests. Cut-off levels: DS VI=-3.69, DS VII=-3.61, DS VII=-2.75, DS $I X=-2.45$, DS XII $=D S$ values between -3.69 and -2.45 excluded, $n=130$ patients.

\begin{tabular}{|c|c|c|c|c|c|c|}
\hline Diagnostic score (DS) & Positive endpoint & Negative endpoint & $\mathrm{TP}$ & FN & FP & $\mathrm{TN}$ \\
\hline 1. Logistic model $\mathrm{DS}_{\mathrm{LP}+} \mathrm{VII}$ & ASBO & Other cause of abdominal pain & 52 & 2 & 187 & 1,087 \\
\hline 2. Logistic model $\mathrm{DS}_{\mathrm{LP}+} \mathrm{VIII}$ & ASBO & Other cause of abdominal pain & 47 & 7 & 71 & 1,203 \\
\hline 3. Logistic model $\mathrm{DS}_{\mathrm{LP}+} \mathrm{IX}$ & ASBO & Other cause of abdominal pain & 50 & 4 & 136 & 1,138 \\
\hline 4. Logistic model $\mathrm{DS}_{\mathrm{LP}+} \mathrm{X}$ & ASBO & Other cause of abdominal pain & 47 & 7 & 62 & 1,212 \\
\hline 5. Logistic model $\mathrm{DS}_{\mathrm{LP}+} \mathrm{XI}$ & ASBO & Other cause of abdominal pain & 47 & 2 & 62 & 1,087 \\
\hline
\end{tabular}

TP: True positive; FN: false negative; FP: false positive; TN: true negative. Logistic regression analysis formula for DS: $1.03 \times 1$ location of pain at diagnosis (positive endpoint $=1$, negative endpoint $=0$ ) $+0.87 \times$ type of pain (positive endpoint $=1$, negative endpoint $=0$ ) $+2.50 \times$ previous abdominal surgery (positive endpoint $=1$, negative endpoint $=0)+2.57 \times$ distension (positive endpoint $=1$, negative endpoint $=0)+2.20 \times$ bowel sounds $($ positive endpoint=1, negative endpoint=0) -7.22 . 


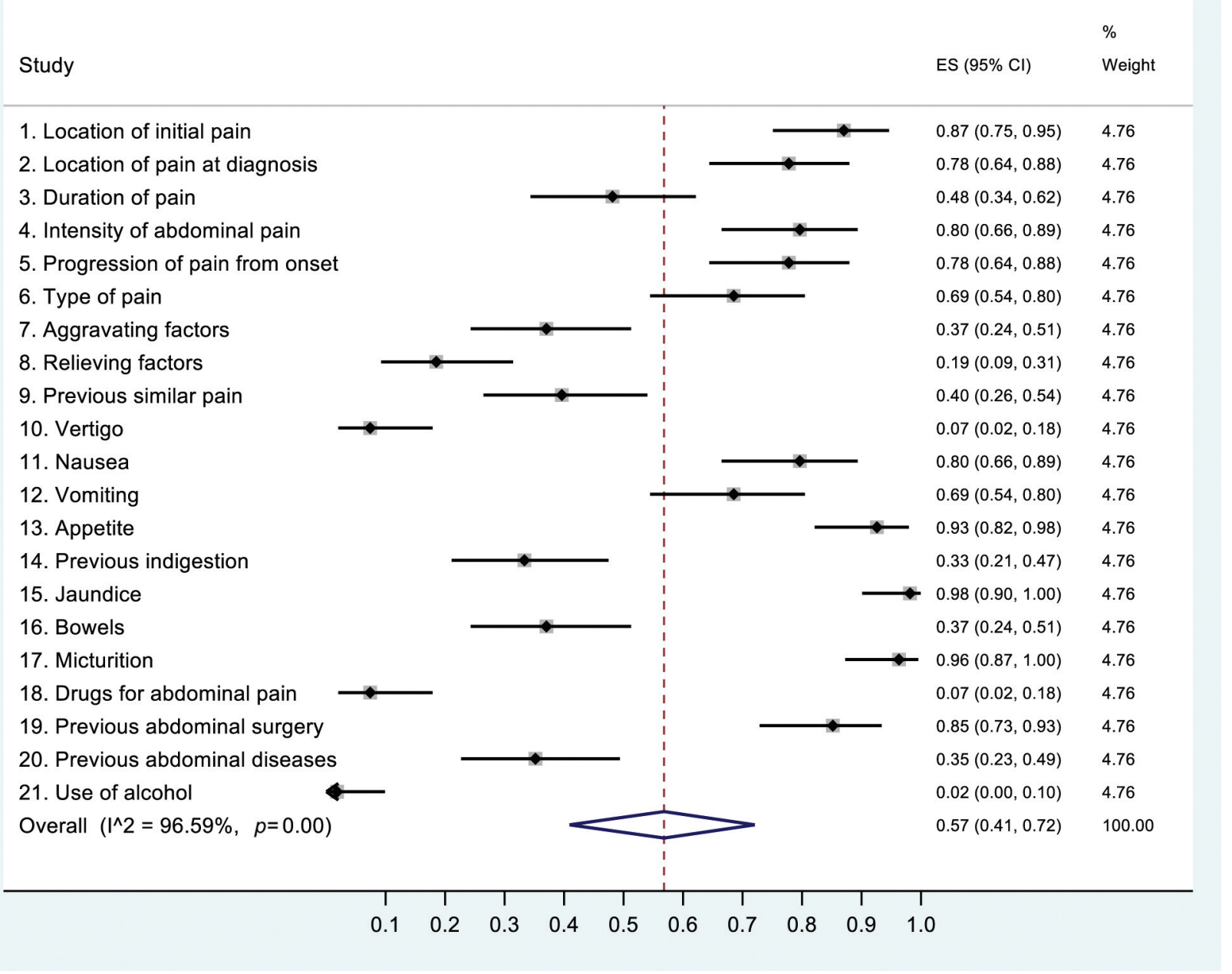

Figure 1. Sensitivities of the history-taking in acute small bowel obstruction (ASBO) (random-effects model). ES: Estimated sensitivity; CI: confidence interval.

positive, urine) showed $85-100 \%$ Se (Figure 3). The overall Sp of the findings was $47 \%(95 \% \mathrm{CI}=30-65 \%)$ (Figure 4$)$, while 8 findings showed $\mathrm{Sp}$ higher than $47 \%$. The five most accurate findings (mood, abdominal movement, scar, distension, bowel sounds) showed 77-96\% Sp (Figure 4).

The DS without location of pain $\left(D S_{L P-}\right)$ in $A S B O$. The most important predictors of ASBO without LP at diagnosis were type of pain, previous abdominal surgery, distension and bowel sounds. The best diagnostic level for the $\mathrm{DS}_{\mathrm{LP}-}$ formula ( $\mathrm{DS} \mathrm{V} ; \mathrm{Se}=93 \%, \mathrm{~S} p=95 \%$ ) was reached when the patients with $\mathrm{DS}_{\mathrm{LP}}$ - values between -3.85 and -1.60 , were considered as "undefined" patients for whom follow-up is required $(n=188)$ (Figure 5). The DS model was tested at five different cut-off levels to disclose the best diagnostic performance (Figure 5). The Se and Sp of these five $\mathrm{DS}_{\mathrm{LP}}$ formulas were $89 \%(95 \% \mathrm{CI}=84-94 \%)$ and $91 \%(95 \% \mathrm{CI}=85-96 \%)$, respectively (Figures 5 and 6$)$. Three of these formulas showed $\mathrm{Se}>89 \%$ and three formulas had $\mathrm{Sp}>91 \%$. The best diagnostic $\mathrm{DS}_{\mathrm{LP}}$ formula in these ASBO patients (formula DS V, Figures 5 and 6) showed $\mathrm{Se}$ of $93 \%(95 \% \mathrm{CI}=82-99 \%)$ and $\mathrm{Sp}$ of $95 \%$ $(95 \% \mathrm{CI}=94-96 \%)$.

The DS with location of pain $\left(D S_{L P+}\right)$ in $A S B O$. The significant independent predictors (LP at diagnosis, type of pain, previous abdominal surgery, distension, bowel sounds) were used to build up the five different $\mathrm{DS}_{\mathrm{LP}+}$ models. The $\mathrm{Se}$ and $\mathrm{Sp}$ of these five $\mathrm{DS}_{\mathrm{LP}+}$ models were $92 \%$ $(95 \% \mathrm{CI}=88-96 \%)$ and $92 \%(95 \% \mathrm{CI}=88-95 \%)$, respectively (Figures 7 and 8). Three formulas showed $\mathrm{Se}>92 \%$ and three formulas $\mathrm{Sp}$ over $92 \%$. The $\mathrm{DS}_{\mathrm{LP}+}$ (formula DS V, 


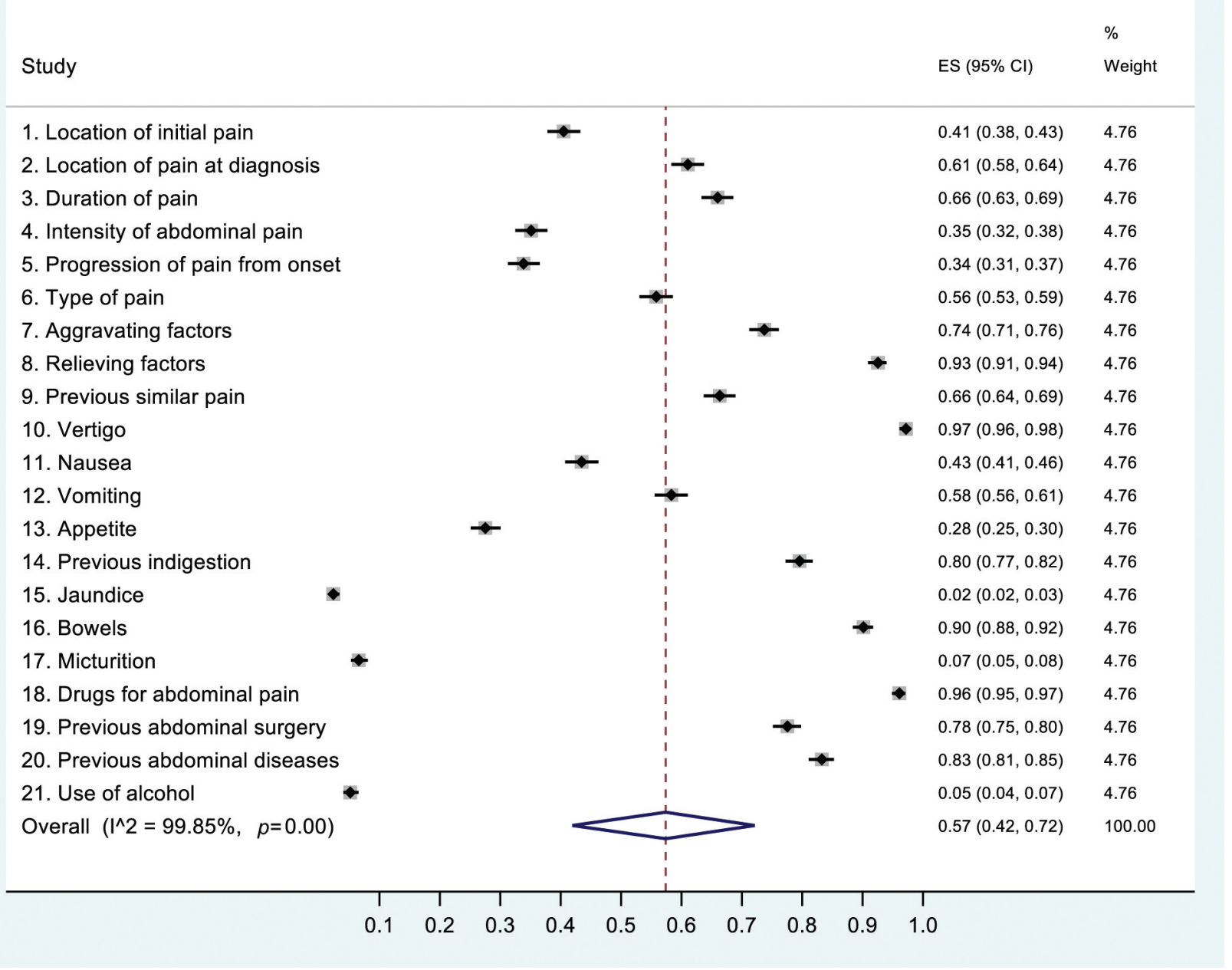

Figure 2. Specificities of the history-taking in acute small bowel obstruction (ASBO) (random-effects model). ES: Estimated specificity; CI: confidence interval.

Figures 7 and 8$)$ showed Se of $96 \%(95 \% \mathrm{CI}=86-100 \%)$ and Sp of $95 \%(95 \% \mathrm{CI}=93-96 \%)$ (Figures 7 and 8$)$.

HSROC and comparison of the AUC values. STATA (metandiplot) was used to draw the HSROC curves to visualise the pooled overall diagnostic performance of the history-taking, findings and different DS formulas in detecting ASBO (Figures 9, 10, 11 and 12). In SROC analysis, the AUC values for i) clinical history-taking, ii) diagnostic findings and tests, iii) $\mathrm{DS}_{\mathrm{LP}_{-}}$and iv) $\mathrm{DS}_{\mathrm{LP}+}$ were as follows: i) $\mathrm{AUC}=0.638 \quad(95 \% \mathrm{CI}=0.600-0.676)$; ii $)$ $\mathrm{AUC}=0.694 \quad(95 \% \mathrm{CI}=0.630-0.724), \quad$ iii $) \quad \mathrm{AUC}=0.962$ (95\% CI $=0.940-0.986)$, and for iv) $\mathrm{AUC}=0.971$ (95\% CI $=0.952-0.988)$. In roccomp analysis for the AUC values, the differences were significant as follows: between i) and ii) $p=0.312$; between i) and iii) $p<0.0001$; between i) and iv) $p<0.0001$; between ii) and iii) $p<0.0001$; between ii) and iv) $p<0.0001$; and between iii) and iv) $p=0.317$.

\section{Discussion}

ASBO requires prompt recognition and early intervention. The decision to operate the patient with suspected ASBO is based on doctors' clinical judgement. The lack of commonly accepted guidelines and diagnostic scoring (DS) for ASBO (1) encouraged us to evaluate the accuracy of the clinical diagnosis of ASBO among AAP patients. The DS models published so far are based on clinicoradiological formulas which need computer tomography (CT) imaging in scoring. Jones et al. (8), in a retrospective study of ASBO patients, correlated CT scoring with the actual treatment and reported that dilated small bowel or free fluid in CT predicted ASBO 


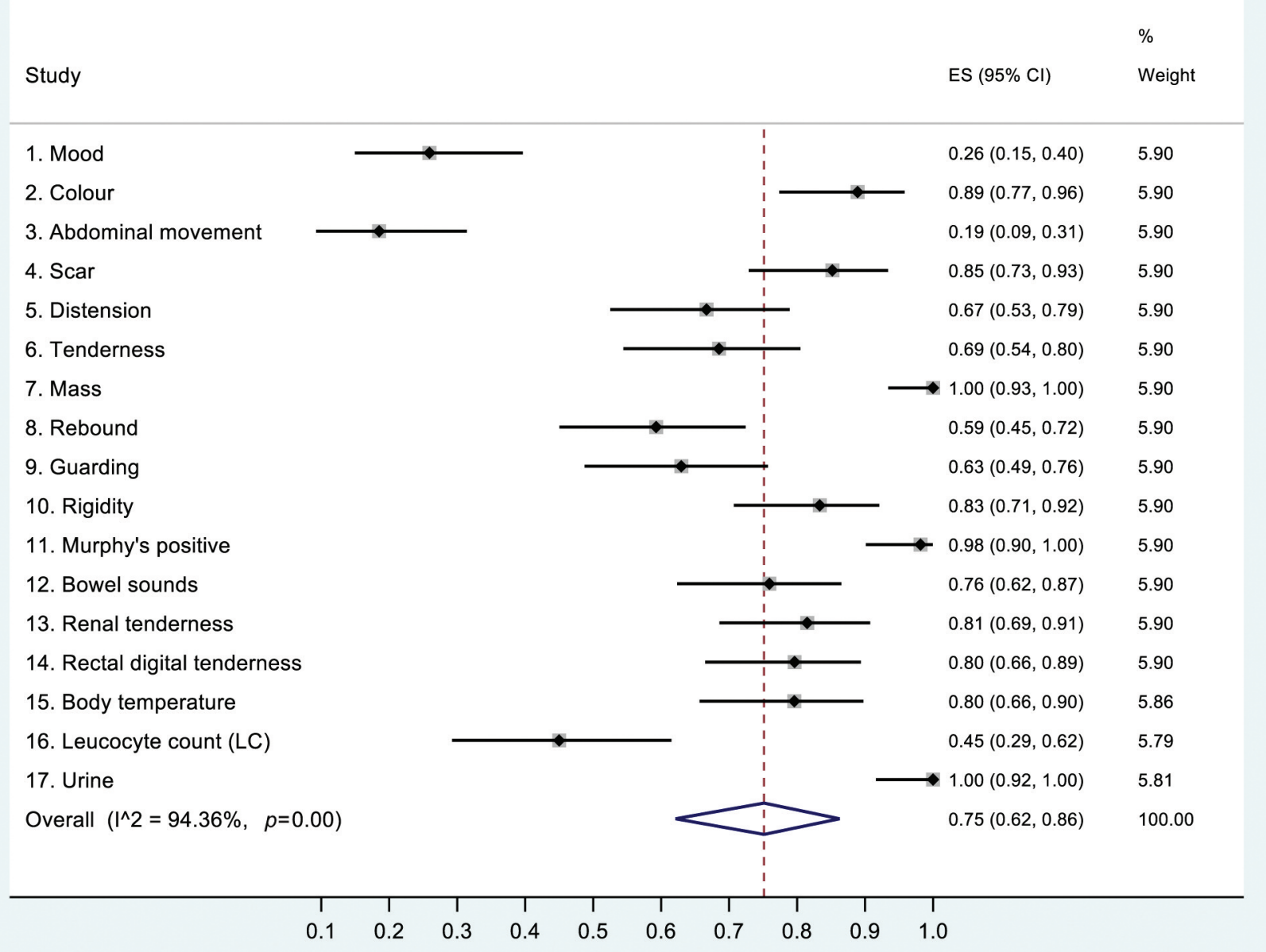

Figure 3. Sensitivities of the signs and tests in acute small bowel obstruction (ASBO) (random-effects model). ES: Estimated sensitivity; CI: confidence interval.

and their DS predicted the need for surgery in $75 \%$ of the patients. However, no ROC analysis with AUC values for the ASBO patients were reported in their study.

Schwenter et al. (9) applied a logistic regression model to construct a DS that would predict ASBO patients. Six variables correlated with ASBO and were given one point each: history of pain, guarding, C-reactive protein level $\geq 75$ $\mathrm{mg} / \mathrm{l}$, leucocyte count (LC) $>10,000 / \mathrm{mm}^{3}$, ascites volume $\geq 500 \mathrm{ml}$ on $\mathrm{CT}$ and reduction of $\mathrm{CT}$ small bowel wall contrast enhancement. A DS $\geq 3$ had $\mathrm{Se}$ of $67.7 \%$ and $\mathrm{Sp}$ $90.8 \%$, with the AUC of 0.87 (95\% CI=0.79-0.95). Zielinski et al. (10) reviewed retrospectively 100 ASBO patients who had undergone CT. The statistically significant predictors of ASBO in multivariate analysis were mesenteric edema, ascites and lacking of "small bowel feces sign" in CT imaging. Their clinicoradiological DS had Se of $96 \%$ and a positive predictive value (PPV) of $90 \% \quad(\mathrm{OR}=16.4$,
95\% CI=3.6-75.4) for ASBO. However, no ROC analysis was performed for the ASBO patients.

Huang et al. (11) analyzed retrospectively a cohort of ASBO patients and in multivariate analysis, $\operatorname{Tax} \geq 38.0^{\circ} \mathrm{C}$, sign of peritoneal irritation, LC $>10,000 \mathrm{~m}^{3}$, CT signs (thickwalled small bowel $\geq 3 \mathrm{~mm}$ ), and ascites were significantly associated with ASBO, with AUC for their DS model reaching 0.935. Bouassida et al. (12) conducted a retrospective study including 124 ASBO patients. In logistic regression analysis, six independent predictive factors of ASBO were identified: age, pain duration, Tax, LC and CT signs of reduced wall enhancement and segmental mesenteric fluid. The AUC value of their clinicoradiological score was 0.92. In a retrospective study of 97 patients with ASBO, Ozawa et al. (13) used multivariate analysis, serum lactate levels, ascites, and peritoneal irritation sign in CT and generated a DS, which showed $92 \%$ Se and $75 \%$ Sp for 


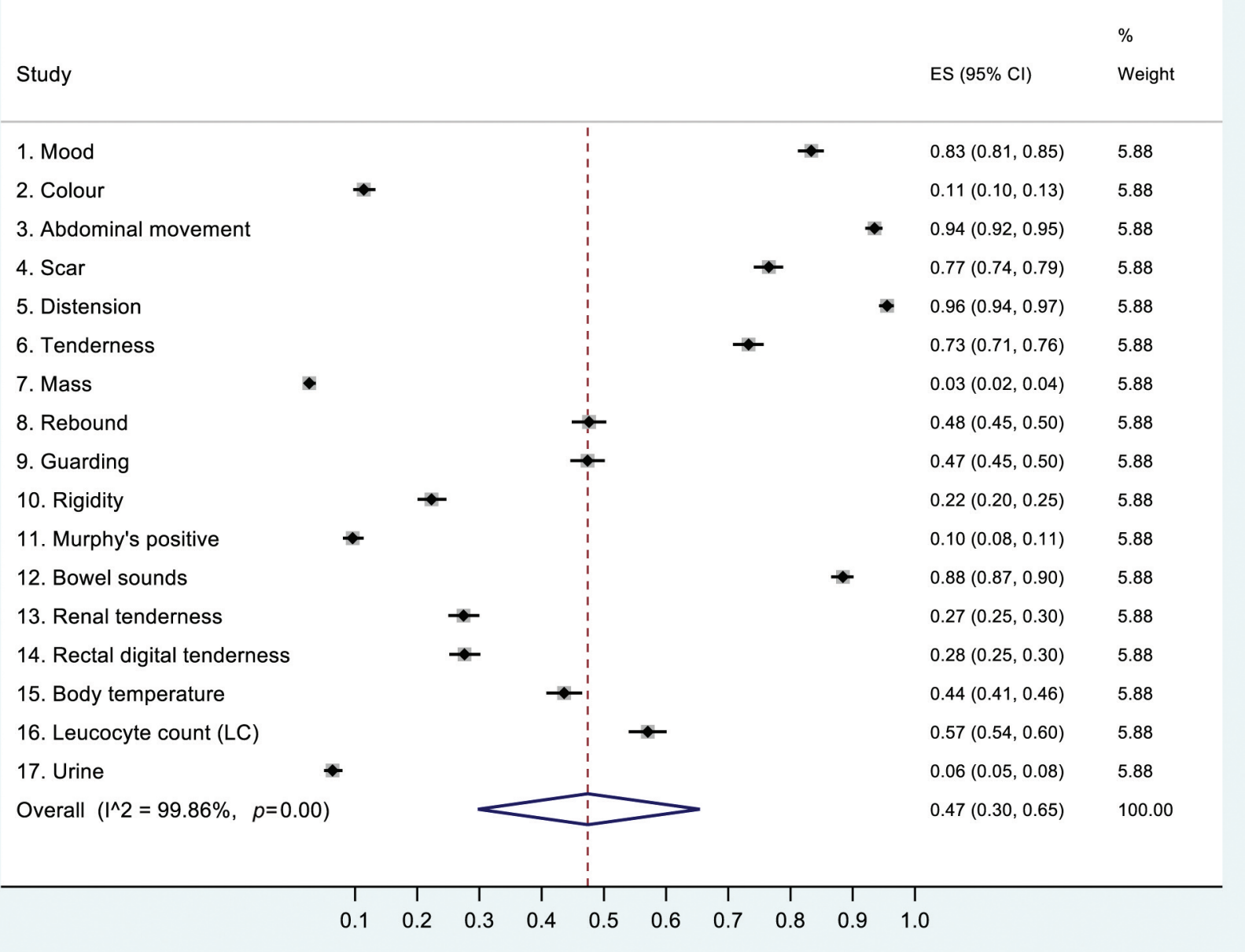

Figure 4. Specificities of the clinical signs and tests in acute small bowel obstruction (ASBO) (random-effects model). ES: Estimated specificity; CI: confidence interval.

ASBO diagnosis. Unfortunately, no ROC analysis was available.

Our interest was to compare the performance of the clinical history-taking variables, clinical findings and laboratory tests to discriminate ASBO patients from those of AA and NSAP patients reported in our previous study (4), to determine whether common clinical variables differ in ASBO and AA/NSAP patients. The overall Se of the history-taking in $\operatorname{ASBO}(57 \%, 95 \% \mathrm{CI}=41-72 \%)$, significantly lower than that detecting AA among NSAP patients $(75 \%, 95 \% \mathrm{CI}=60$ $87 \%)$. In contrast, the $\mathrm{Sp}$ of the clinical history-taking in diagnosis of $\mathrm{ASBO}(57 \%, 95 \% \mathrm{CI}=42-72 \%)$ was significantly higher than that in AA/NSAP patients $(35 \%, 95 \% \mathrm{CI}=23-$ $49 \%)$. Instead, the Se of the clinical findings and laboratory tests in ASBO $(75 \%, 95 \% \mathrm{CI}=62-86 \%)$ was lower than that among AA/NSAP patients $(87 \%, 95 \% \mathrm{CI}=81-92 \%)$. However, the $\mathrm{Sp}$ of the clinical findings and tests in diagnosis of ASBO $(47 \%, 95 \% \mathrm{CI}=30-65 \%)$ was higher than that of the AA/NSAP patients $(38 \%, 95 \% \mathrm{CI}=19-59 \%)$.

When the performance of the DS models was compared between ASBO and AA/NSAP patients, the trend was similar. The Se of the DS models in diagnosis of ASBO $(92 \%, 95 \% \mathrm{CI}=88-96 \%)$ was significantly higher than that in AA/NSAP patients $(79 \%, 95 \% \mathrm{CI}=72-85 \%)$. Although, Se and $\mathrm{Sp}$ usually behave reciprocally, it was a surprise to find that the Sp of the DS in ASBO patients $(92 \%, 95 \% \mathrm{CI}=88$ $95 \%)$ was similar to that in the AA/NSAP patients $(95 \%$, 95\% CI=93-97\%).

ASBO patients $D S_{L P-}$ and $D S_{L P+}$. AUC values based on HSROC comparison test showed that the diagnostic performance of the clinical findings was slightly better than that of the clinical history-taking details only $(p=0.312)$. However, as measured by the AUC values, the DS model was superior to both i) the clinical history-taking and ii) findings and tests. 


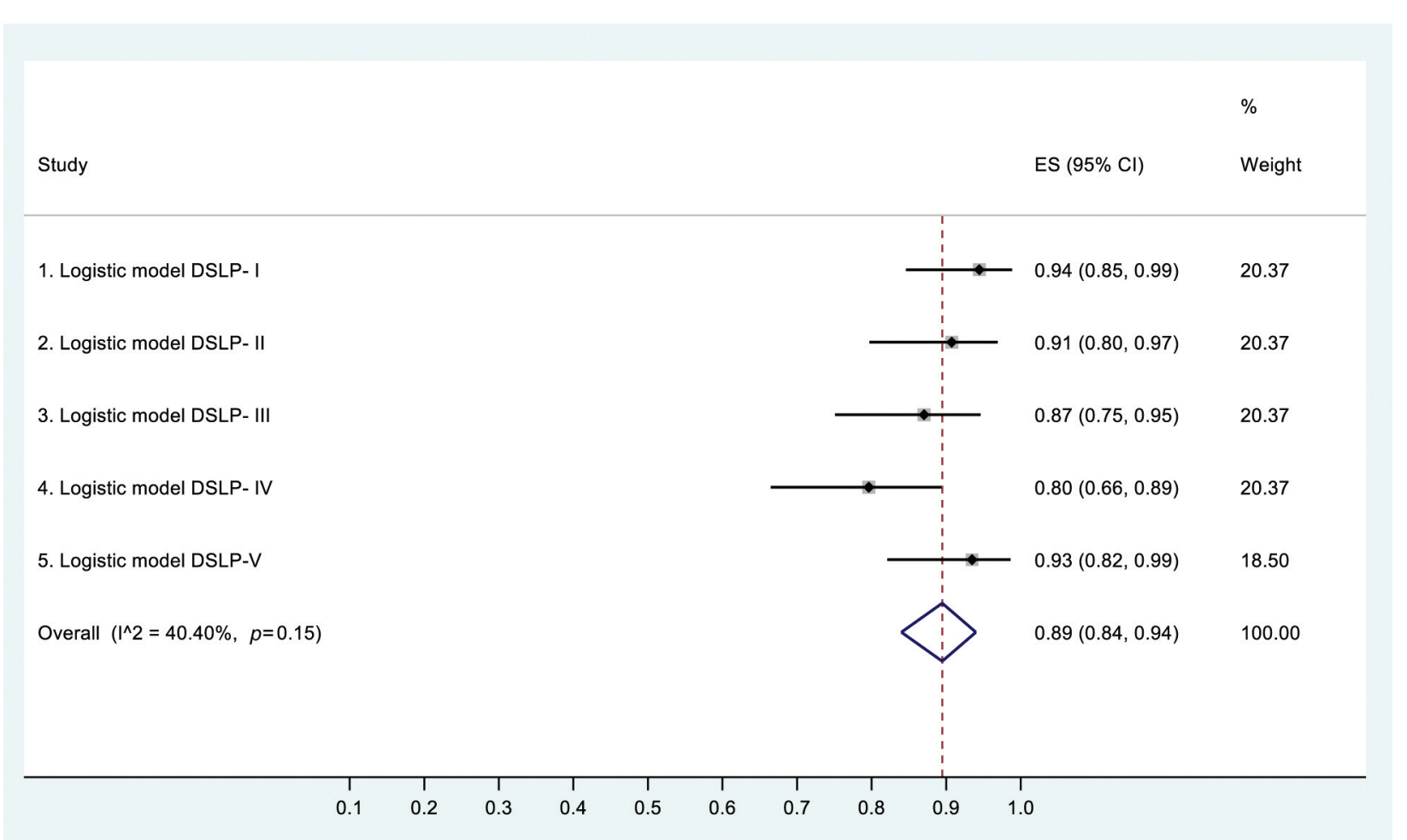

Figure 5. Sensitivities of diagnostic scores without location of pain $\left(D S_{L P-}\right)$ at five different cut-off levels (DS I-V). ES: Estimated sensitivity; CI: confidence interval.

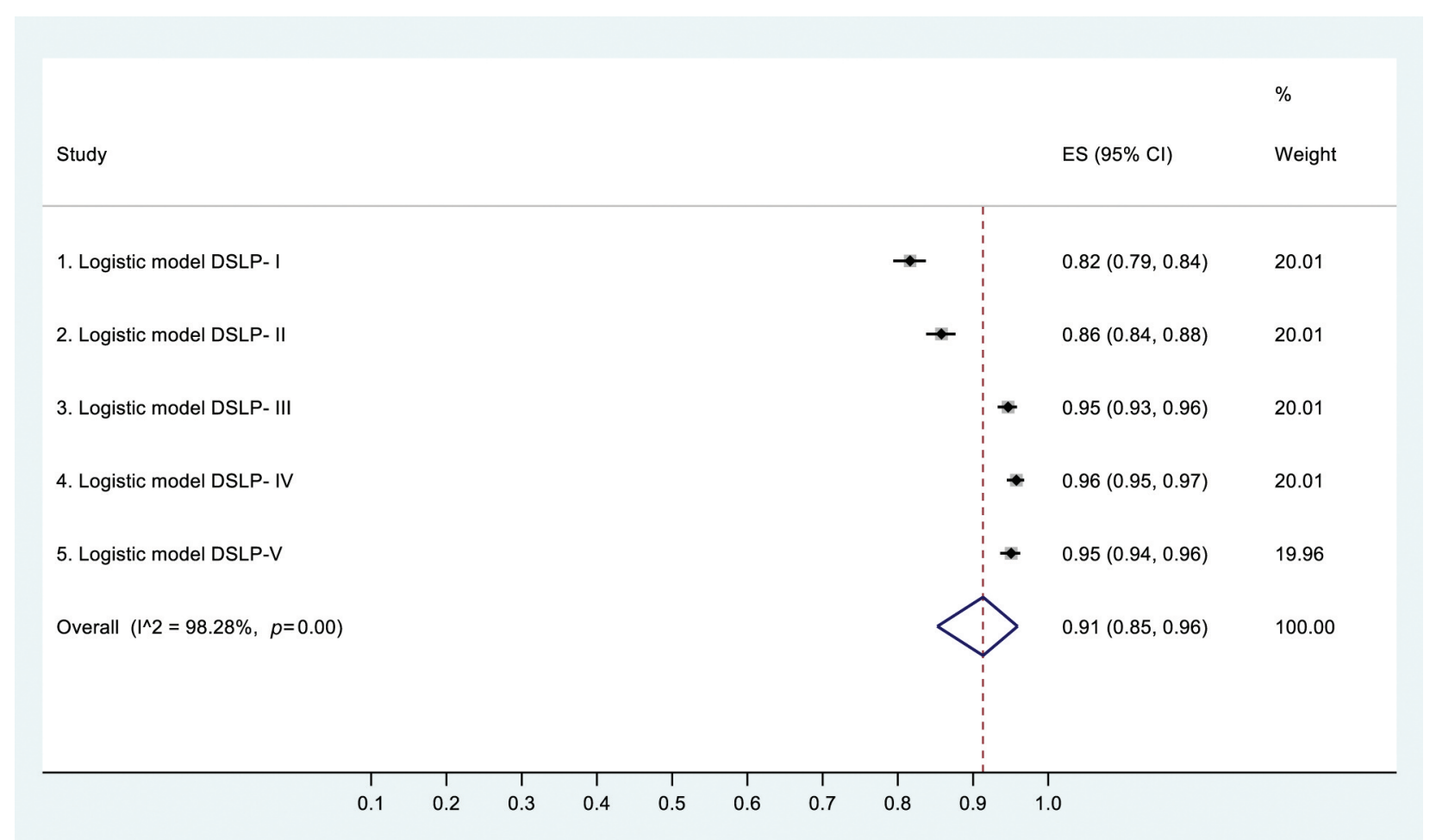

Figure 6. Specificities of diagnostic scores without location of pain $\left(D S_{L P \_}\right)$at five different cut-off levels (DS I-V). ES: Estimated specificity; CI: confidence interval. 


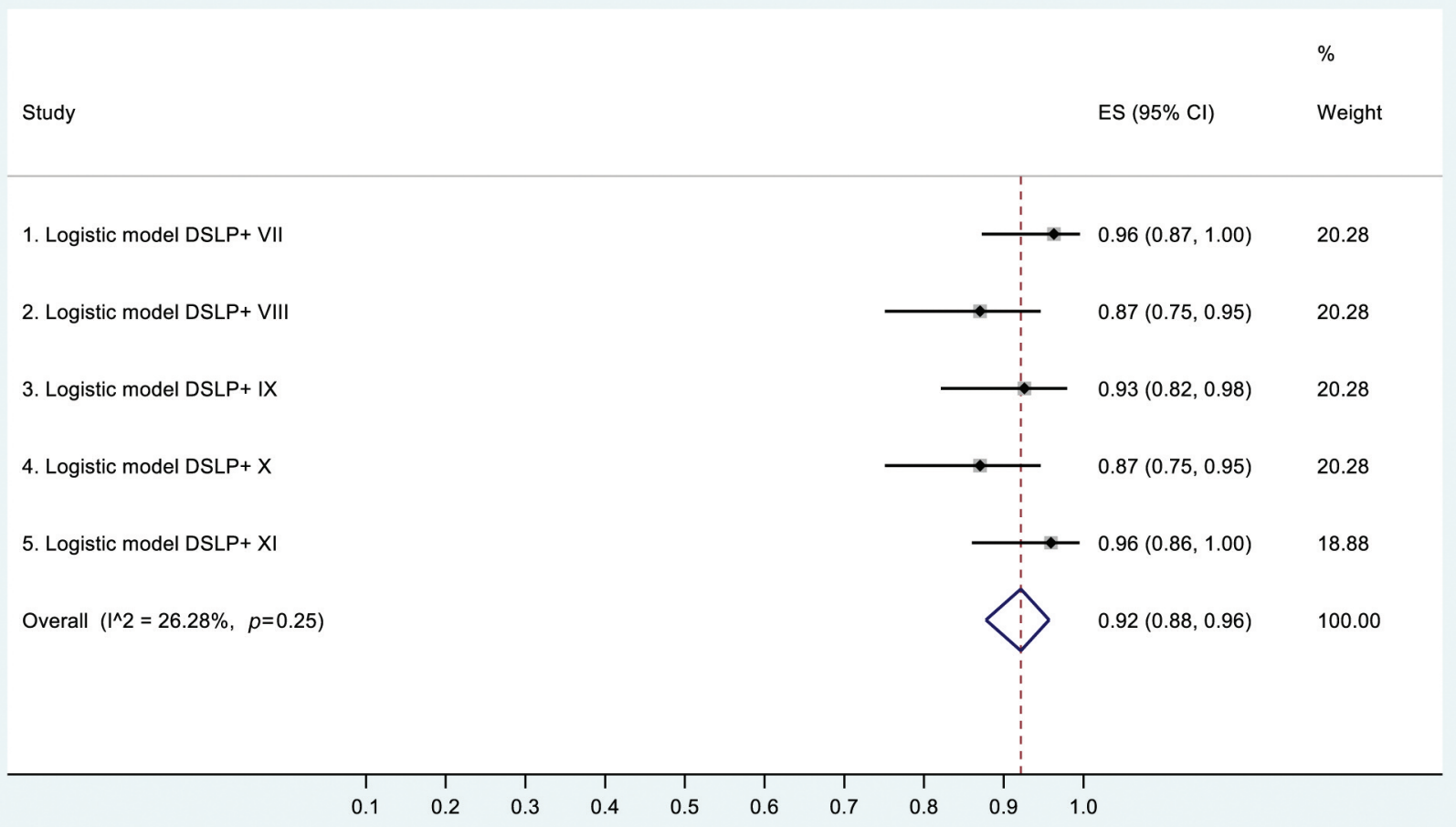

Figure 7. Sensitivities of diagnostic scores with location of pain $\left(D S_{L P+}\right)$ at five different cut-off levels (DS VI-X). ES: Estimated sensitivity; CI: confidence interval.

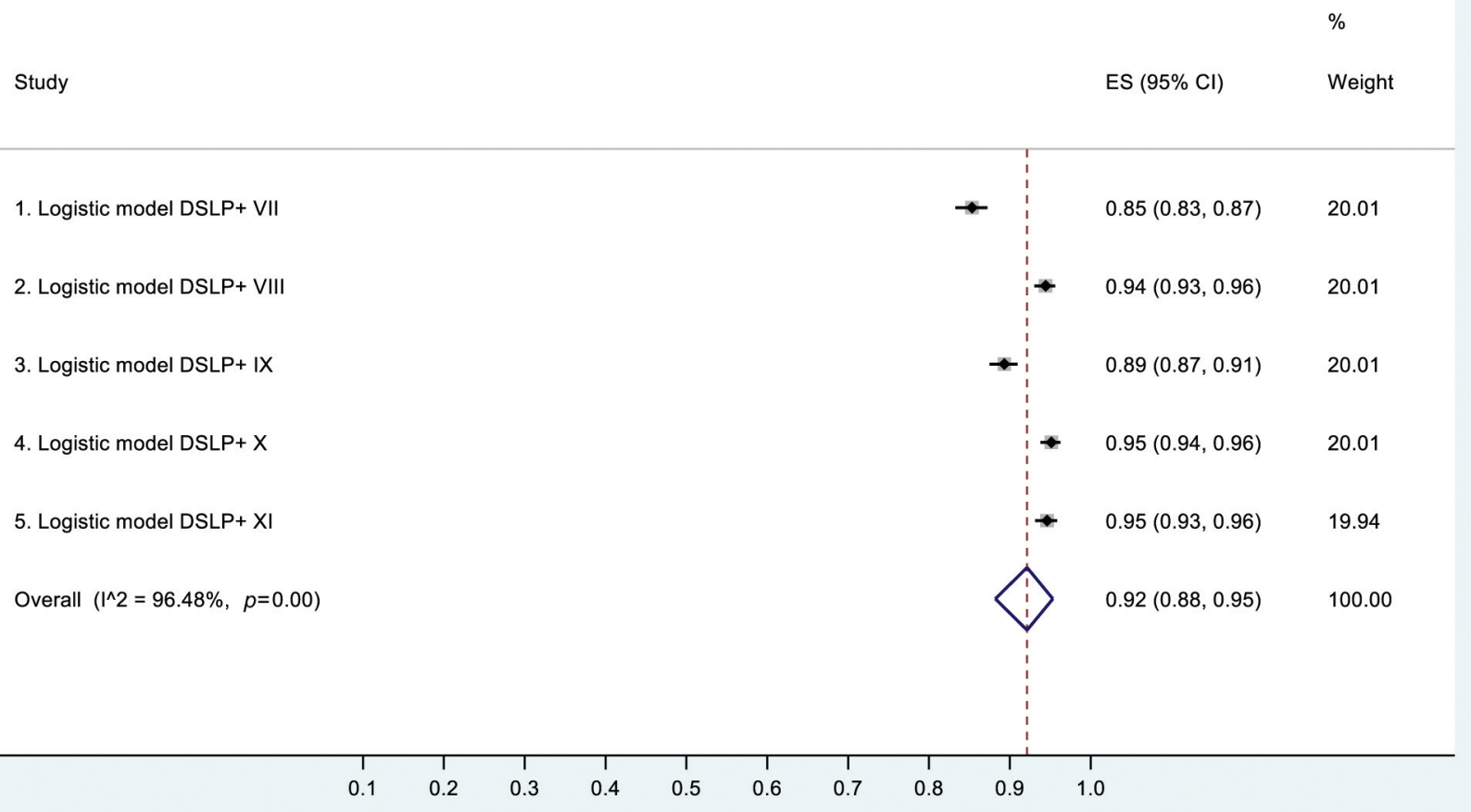

Figure 8. Specificities of diagnostic scores with location of pain $\left(D S_{L P+}\right)$ at five different cut-off levels (DS IV-X). ES: Estimated specificity; CI: confidence interval. 


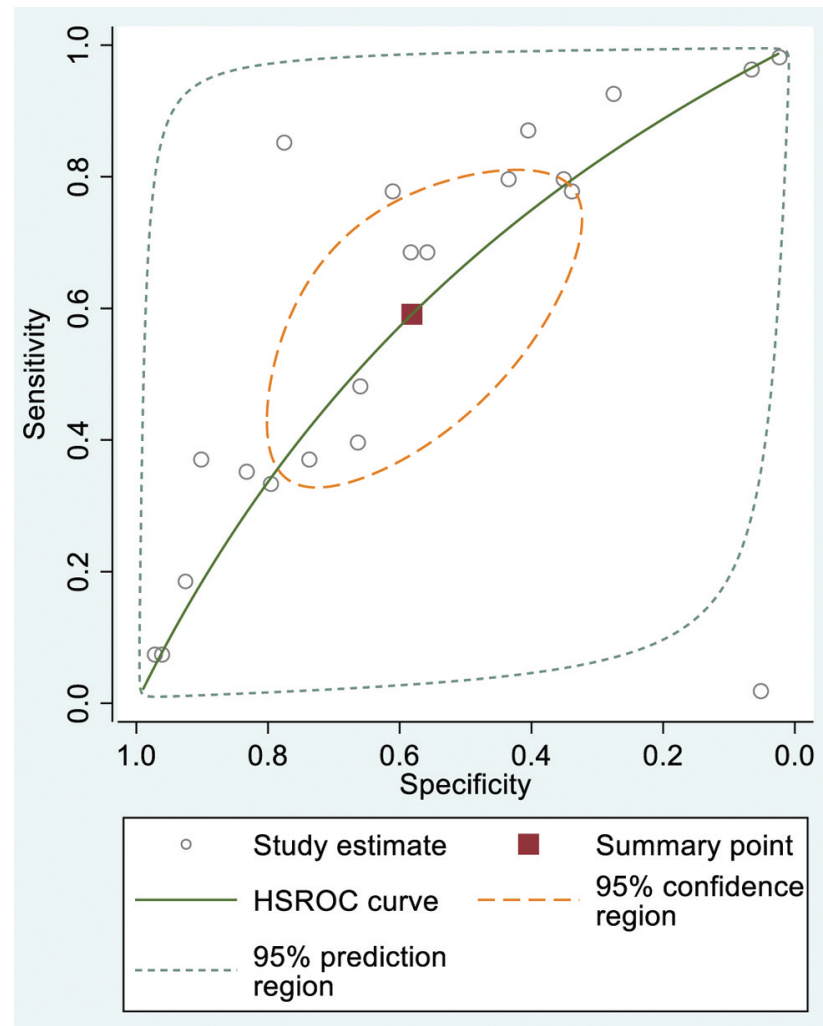

Figure 9. Hierarchical summary receiver operating characteristic (HSROC) curve of the history-taking in acute small bowel obstruction $(A S B O)$.

Previous studies with a design similar to ours are scanty and only few retrospective studies with CT imaging have evaluated the applicability of the DS models in the ASBO population. Some previous studies have found a significant correlation between history of pain and $\operatorname{ASBO}(9,12)$, but in the present study, the AUC value of the $D S_{L P-}$ in ASBO patients (AUC $=0.962,95 \% \mathrm{CI}=0.940-0.986)$ was almost identical with that in the $D S_{L P+}$ patients $(\mathrm{AUC}=0.971 .95 \% \mathrm{CI}=0.952-0.988)$.

\section{Conclusion}

As far as we know, there is paucity of DS models based on clinical findings and laboratory tests. Our results indicate that DS based on clinical findings could be an important part of the diagnostic algorithm, because the doctor's decision to operate patients with ASBO is based on clinical judgement (1). However, even with radiological and laboratory analyses (14-16), it may be difficult to reach a higher efficiency than the $97 \%$ AUC (Se/Sp balance) for the DS in ASBO. To date, the only DS study of patients with ASBO has not yet been closed and analyzed (17) and the present study is the first to provide data that the DS could be used for detection of

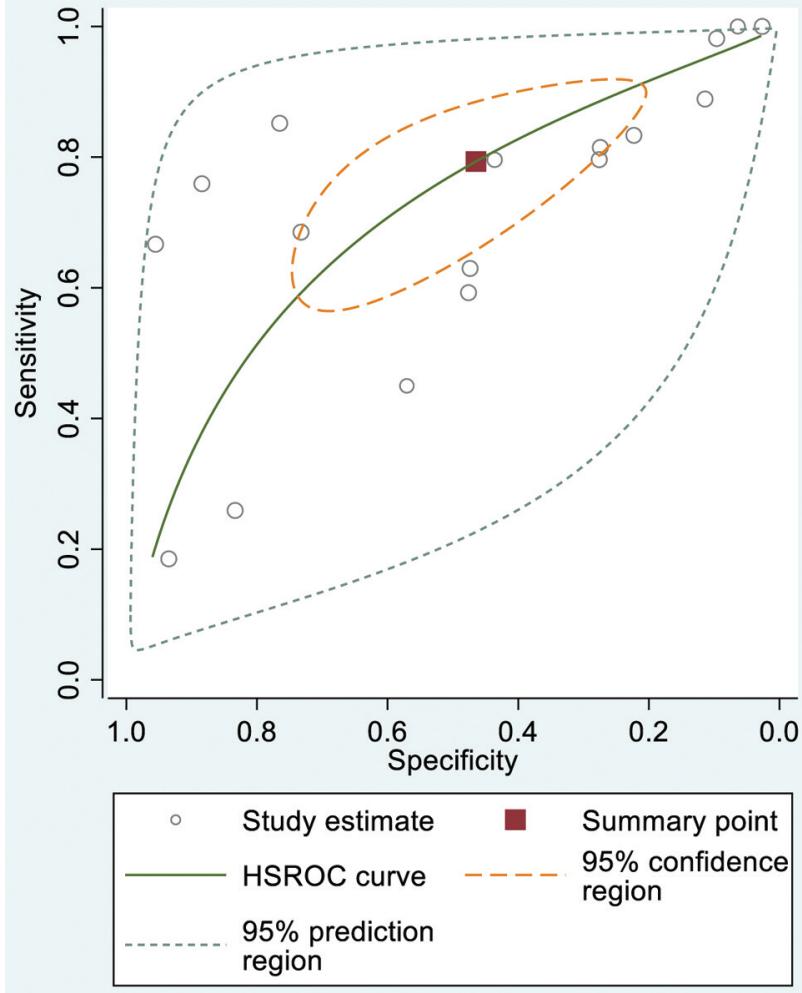

Figure 10. Hierarchical summary receiver operating characteristic (HSROC) curve of the clinical signs and tests in acute small bowel obstruction (ASBO).

ASBO without radiological or laboratory analyses to reach a high diagnostic accuracy in AAP patients.

\section{Conflicts of Interest}

The Authors have no conflicts of interest or financial ties to disclose in relation to this study.

\section{Authors' Contributions}

All Authors contributed to the collection and analysis of data, drafting and revising the manuscript, read and approved the final manuscript.

\section{Acknowledgements}

The study was funded by the Päivikki and Sakari Sohlberg Foundation.

\section{References}

1 Ten Broek RPG, Krielen P, Di Saverio S, Coccolini F, Biffl WL, Ansaloni L, Velmahos GC, Sartelli M, Fraga GP, Kelly MD, Moore FA, Peitzman AB, Leppaniemi A, Moore EE, Jeekel J, Kluger Y, 


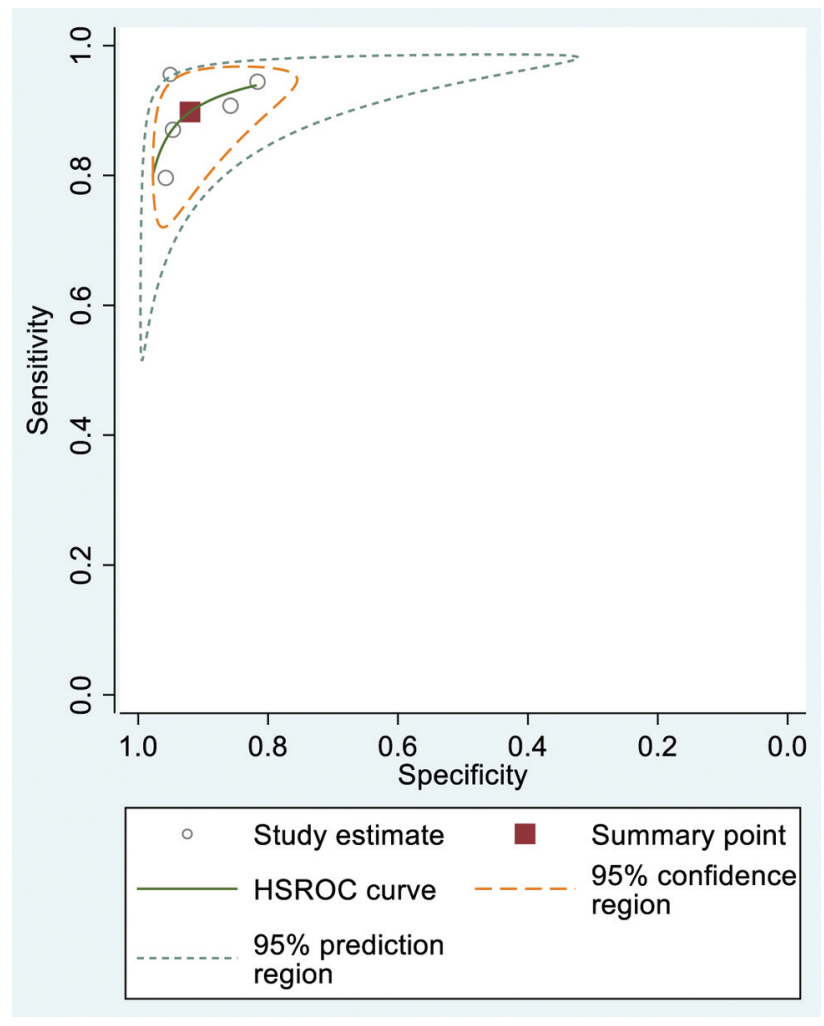

Figure 11. Hierarchical summary receiver operating characteristic (HSROC) curve of the five $\left(D S_{L P-}\right)$.

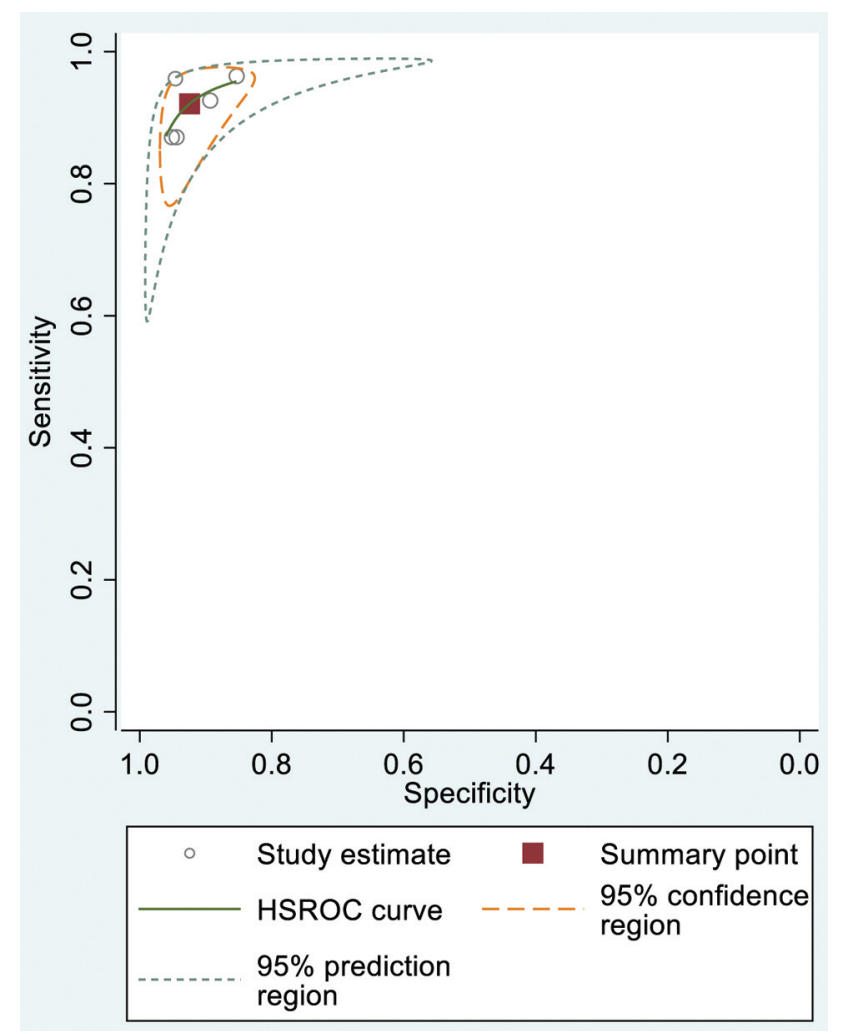

Figure 12. Hierarchical summary receiver operating characteristic (HSROC) curve of the five $\left(D S_{L P+}\right)$.
Sugrue M, Balogh ZJ, Bendinelli C, Civil I, Coimbra R, De Moya M, Ferrada P, Inaba K, Ivatury R, Latifi R, Kashuk JL, Kirkpatrick AW, Maier R, Rizoli S, Sakakushev B, Scalea T, Søreide K, Weber D, Wani I, Abu-Zidan FM, De'Angelis N, Piscioneri F, Galante JM, Catena $\mathrm{F}$ and van Goor $\mathrm{H}$ : Bologna guidelines for diagnosis and management of adhesive small bowel obstruction (ASBO): 2017 update of the evidence-based guidelines from the world society of emergency surgery ASBO working group. World J Emerg Surg 13: 24, 2018. PMID: 29946347. DOI: 10.1186/s13017-018-0185-2

2 Parker M, Ellis H, Moran B, Thompson J, Wilson M, Menzies D, Mcguire A, Lower A, Hawthorn R, O'brien F, Buchan S and Crowe A: Postoperative adhesions. Diseases of the Colon \& Rectum 44(6): 822-829, 2021. DOI: 10.1007/BF02234701

3 Eskelinen $\mathrm{M}$ and Lipponen P: Usefulness of history-taking in non-specific abdominal pain: a prospective study of 1333 patients with acute abdominal pain in Finland. In Vivo 26(2): 335-339, 2012. PMID: 22351680.

4 Meklin J, Eskelinen M, Syrjänen K and Eskelinen M: Leucocyte count does not improve the diagnostic performance of a Diagnostic Score (DS) in distinguishing Acute Appendicitis (AA) from Nonspecific Abdominal Pain (NSAP). In Vivo 34(6): 3327-3339, 2020. PMID: 33144440 . DOI: 10.21873 /invivo.12171

5 Meklin J, Eskelinen M, Syrjänen K and Eskelinen M: Genderspecific performance of a diagnostic score in Acute Appendicitis.
In Vivo 34(6): 3687-3703, 2020. PMID: 33144486. DOI: 10.21873/invivo.12217

6 Eskelinen M, Meklin J, Syrjänen K and Eskelinen M: Performance of a diagnostic score in confirming Acute Cholecystitis among patients with acute abdominal pain. Anticancer Res 40(12): 69476956, 2020. PMID: 33288589. DOI: 10.21873/anticanres.14719

7 Eskelinen M, Meklin J, Syrjänen K and Eskelinen M: Pediatric acute appendicitis score in children with Acute Abdominal Pain (AAP). Anticancer Res 41(1): 297-306, 2021. PMID: 33419824. DOI: $10.21873 /$ anticanres.14776

8 Jones K, Mangram AJ, Lebron RA, Nadalo L and Dunn E: Can a computed tomography scoring system predict the need for surgery in small-bowel obstruction? Am J Surg 194(6): 780-783; discussion 783-784, 2007. PMID: 18005771. DOI: 10.1016/ j.amjsurg.2007.09.020

9 Schwenter F, Poletti PA, Platon A, Perneger T, Morel P and Gervaz P: Clinicoradiological score for predicting the risk of strangulated small bowel obstruction. Br J Surg 97(7): 11191125, 2010. PMID: 20632281. DOI: 10.1002/bjs.7037

10 Zielinski MD, Eiken PW, Bannon MP, Heller SF, Lohse CM, Huebner M and Sarr MG: Small bowel obstruction-who needs an operation? A multivariate prediction model. World J Surg 34(5): 910-919, 2010. PMID: 20217412. DOI: 10.1007/s00268010-0479-3 
11 Huang X, Fang G, Lin J, Xu K, Shi H and Zhuang L: A prediction model for recognizing strangulated small bowel obstruction. Gastroenterol Res Pract 2018: 7164648, 2018. PMID: 29780412. DOI: 10.1155/2018/7164648

12 Bouassida M, Laamiri G, Zribi S, Slama H, Mroua B, Sassi S, Aboudi R, Mighri MM, Bouzeidi K and Touinsi H: Predicting intestinal ischaemia in patients with adhesive small bowel obstruction: A simple score. World J Surg 44(5): 1444-1449, 2020. PMID: 31925521. DOI: 10.1007/s00268-020-05377-6

13 Ozawa M, Ishibe A, Suwa Y, Nakagawa K, Momiyama M, Watanabe J, Yamagishi S, Kubota K and Endo I: A novel discriminant formula for the prompt diagnosis of strangulated bowel obstruction. Surg Today: 2021. PMID: 33420825. DOI: 10.1007/s00595-020-02213-1

14 Markogiannakis H, Memos N, Messaris E, Dardamanis D, Larentzakis A, Papanikolaou D, Zografos GC and Manouras A: Predictive value of procalcitonin for bowel ischemia and necrosis in bowel obstruction. Surgery 149(3): 394-403, 2011. PMID: 20869092. DOI: 10.1016/j.surg.2010.08.007
15 Netz U, Perry Z, Mizrahi S, Kirshtein B, Czeiger D, Sebbag G, Reshef A and Douvdevani A: Cell-free deoxyribonucleic acid as a prognostic marker of bowel ischemia in patients with small bowel obstruction. Surgery 162(5): 1063-1070, 2017. PMID: 28797546. DOI: 10.1016/j.surg.2017.06.015

16 Purdy M, Kokki M, Anttila M, Aspinen S, Juvonen P, Korhonen R, Selander T, Kokki H and Eskelinen M: Does the rectus sheath block analgesia reduce the inflammatory response biomarkers' IL$1 \mathrm{ra}, \mathrm{IL}-6, \mathrm{IL}-8, \mathrm{IL}-10$ and IL-1 $\beta$ concentrations following surgery? A randomized clinical trial of patients with cancer and benign disease. Anticancer Res 36(6): 3005-3011, 2016. PMID: 27272818.

17 Clinicaltrials gov.Diagnostic scoring for small bowel obstruction. Available at: https://clinicaltrials.gov/ct2/show/NCT03461744 [Last accessed on February 12, 2021]

Received February 16, 2021

Revised March 2, 2021

Accepted March 3, 2021 